\title{
METHODOLOGY
}

\section{Rapid skin anaesthesia using high velocity lignocaine particles: a prospective placebo controlled trial}

\author{
A R Wolf, P A Stoddart, P J Murphy, M Sasada
}

Arch Dis Child 2002;86:309-312

Background: Local anaesthetic creams (EMLA and Ametop) are used widely to provide pain free intravenous cannulation. However, they take a minimum of 45 minutes to become effective.

Aims: To evaluate a prototype device, dermal Powderject lidocaine (DPL), that delivers high velocity lignocaine particles into the skin.

Methods: A total of 132 children (aged 4-12 years) were randomised to receive either a sham delivery or a delivery of DPL on the skin at the antecubital fossa, or back of hand. Pain of intravenous cannulation was assessed four minutes later using self reporting behaviours and blinded observation with standard pain assessment tools. The trial was designed to measure both efficacy of skin anaesthesia and potential skin damage with increasing driving pressure of the device (30 or 40 bar), and different lignocaine particle sizes $(<38 \mu \mathrm{m}$ or $38-53 \mu \mathrm{m})$ in a block randomised fashion.

See end of article for authors' affiliations Results: A total of 128 patients were evaluable. There was a trend towards improved anaesthesia at higher device pressures at the antecubital fossa with both self reporting and blinded observation. Acceptable analgesia was achieved in $90 \%$ of patients for high pressure at both particle sizes

Correspondence to: Prof. A R Wolf, Department of Paediatric Intensive Care, Bristol Royal Children's Hospital, St Michael's Hill, Bristol BS2 8BJ, UK;

awolfbch@aol.com

Accepted for publication 11 December 2001 compared to $60 \%$ and $40 \%$ for the sham device using self reporting measures. The observed differences using the blinded observer were similar: 90\% v 20\% (40 bar and small particles $v$ sham), and $80 \% \vee 40 \%$ (40 bar and large particles $v$ sham). At the back of hand the differences between active and sham devices were not significant. The device was well tolerated and not associated with pain on deployment. One patient had mild petechiae and oedema after deployment (Draize score of 3).

Conclusions: This prototype device appears to provide significant skin anaesthesia at the antecubital fossa, but not at the back of hand. The device is not painful to use and causes only minor short term skin changes.

E ffective skin analgesia has revolutionised paediatric practice, allowing painless venepuncture and intravenous cannulation. The first of these agents, EMLA (a eutectic mixture of lignocaine and prilocaine), has been shown to provide reliable anaesthesia for venepuncture if applied on the skin for 60 to 90 minutes, ${ }^{1}$ but the time to achieve effective analgesia has remained a limitation. Topical amethocaine (Ametop) has a more rapid onset of anaesthesia, ${ }^{2}$ and is more effective than EMLA when using the recommended application times of 45 minutes for Ametop and 60 minutes for EMLA. ${ }^{3}$ However, an onset time of 45 minutes remains a practical barrier to the provision of pain free venous cannulation in all children. One solution is to provide topical cream for the parents to apply before arrival in hospital, ${ }^{4}$ but this is not always feasible. New developments in delivery of drugs directly into the skin may offer a solution. Pressurised helium gas can be used to accelerate particles to velocities sufficient to penetrate the outer skin layers. ${ }^{5}$ Preliminary studies on adult volunteers using this principle (dermal Powderject lignocaine, DPL), found that effective topical anaesthesia can be achieved within three minutes after administration and that this technique could provide surface anaesthesia for venepuncture. We therefore wished to determine if a prototype device could provide effective skin analgesia for venepuncture in unpremedicated children, and if the technique was feasible, pain free, and safe in a clinical situation.

\section{METHODS}

After local ethical approval and informed parental consent, 132 white children (aged 4-12 years of age) due to undergo day case dental surgery under general anaesthesia were

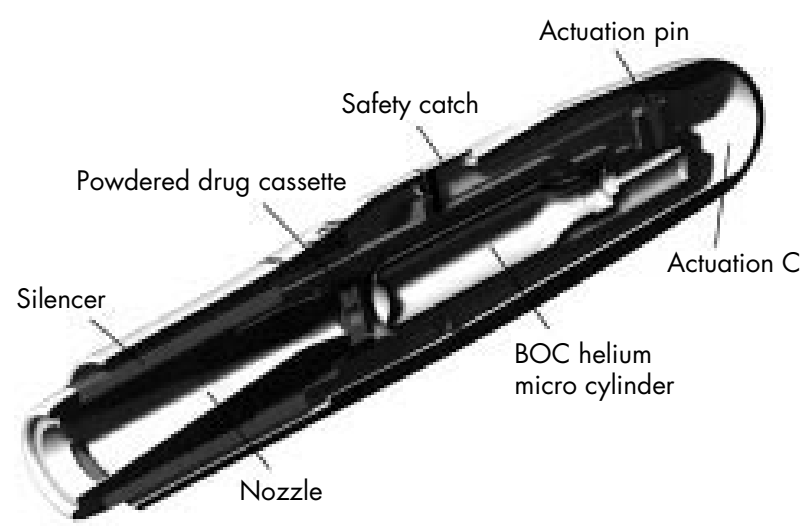

Figure 1 Schematic diagram of Powderject device.

recruited for this randomised, prospective, placebo (sham) controlled trial. The children were randomly allocated to one of two application sites for intravenous access: the back of the hand $(\mathrm{BH})$ or the antecubital fossa (ACF). The DPL delivery system comprises a stainless steel gas cylinder filled with helium under pressure, a stainless steel rupture chamber, a drug cassette, a supersonic nozzle, and a silencer (fig 1). Actuation releases a small volume of compressed helium,

Abbreviations: $\mathrm{ACF}$, antecubital fossa; $\mathrm{BH}$, back of hand; DPL, dermal Powderject lignocaine 
Table 1 Group allocation of activation pressure and lignocaine particle size for DPL

\begin{tabular}{lll}
\hline Study group & $\begin{array}{l}\text { Activation pressure } \\
\text { (bar) }\end{array}$ & $\begin{array}{l}\text { Lignocaine particle } \\
\text { size }(\mu \mathrm{m})\end{array}$ \\
\hline A & 30 & $<38$ \\
B & 30 & $38-53$ \\
C & 40 & $<38$ \\
D & 40 & $38-53$ \\
\hline
\end{tabular}

Patients randomised to sham treatment within the study group received the same activation pressure but did not have lignocaine.

which ruptures the membranes of the cassette and accelerates the powdered lignocaine $(3 \mathrm{mg})$ to high velocities.

In this study we wished to evaluate the relative efficacy of varying both the driving pressure of the helium gas (activation pressure), and the lignocaine particle size. The activation pressures chosen for study were 30 and 40 bar, and the two ranges of lignocaine particle sizes chosen for investigation were $<38 \mu \mathrm{m}$ and $38-53 \mu \mathrm{m}$. Higher activation pressures and particle sizes were considered likely to be more effective but potentially damaging to the skin. Therefore, patients were block randomised into four groups starting with low activation pressures and small lignocaine particle size at each of the two application sites (BH or ACF) and progressing to the higher activation pressures and particle sizes (table 1). Five patients in each group were allocated blindly to receive an identical sham device containing helium gas but with an empty drug cassette.

Three minutes after the device was deployed, venous cannulation was performed using a 22 gauge cannula. All children were evaluated for pain responses immediately after application of DPL and again following intravenous cannulation. Pain and discomfort were evaluated using two self assessment pain scales, the Oucher ${ }^{6}$ and the Faces ${ }^{7}$ scales. The Oucher includes six photographs of a child with varying degrees of pain and a corresponding numerical visual analogue scale, with the child using either the visual or numerical score depending on their cognitive abilities. The Faces consists of six faces representing no pain to maximum pain possible. For the purposes of this study, acceptable anaesthesia on the Oucher scale was predefined as a pain score of 20 or less on the numerical scale or the lowest two corresponding pictures on the photographic scale. In addition, a blinded nurse assessment of the child's pain was made using a four point scale $(0=$ no pain, $1=$ mild pain, $2=$ moderate pain, 3 $=$ severe pain).

Evaluation of the skin effects from DPL activation as increasing pressure and lignocaine particle sizes were used was necessary to determine the safety of the device. The application site was evaluated immediately before and after application of DPL for erythema and oedema using the Draize scale. ${ }^{8}$ This is a numeric scale describing skin trauma varying from 0 (no effect) to 4 (severe erythema and or oedema greater than $1 \mathrm{~mm}$ in depth). A Draize score of 0 to 2 was considered acceptable. Any bleeding at the application site was also recorded. These observations were repeated at 30 and 60 minutes after venous cannulation. Photographs of each application were made at the same time as the nurse observations and were independently monitored. It was predetermined as part of the study design that if any patient was given a Draize score of 4 , the study would immediately be terminated.

\section{Statistics}

A two group $\chi^{2}$ test with a 0.05 two sided significance level has $80 \%$ power to detect a difference in the proportion of patients with acceptable anaesthesia between the sham device (expected to be 20\%) and the active device containing lignocaine hydrochloride (expected to be $90 \%$ ). This results in an odds ratio of 36, when the sample sizes are five sham and 10 active, respectively (a total sample size of 15). Therefore 15 patients within each activation condition and body site (BH or ACF) were randomised to receive either lignocaine hydrochloride or the sham device with an allocation ratio of $2: 1$, resulting in 10 patients randomised to lignocaine and five patients to the sham device.

The Faces pain scale was analysed using analysis of variance. Using the error variance from this, pairwise comparisons between the active and sham devices were conducted using the Student's $t$ distribution, and estimates of the differences in the adjusted means were calculated. The proportion of patients with acceptable anaesthesia on the Oucher scale was analysed separately for activation condition and site of administration (BH or ACF) using logistic regression. Pairwise comparisons between the active and sham devices were conducted using the Wald statistic ${ }^{9}$ and estimates of the odds ratio and $95 \%$ confidence interval were calculated. The proportion of patients assessed by the nurse as having no pain immediately post-cannulation was analysed post hoc using logistic regression and the significance level of the treatment effect investigated using the Wald statistic. A significance level of less than 0.05 was considered to be statistically significant.

\section{RESULTS}

A total of 132 patients were recruited for study of which 128 received the study treatment, the remaining four patients being unable to enter the study after consent because of noncompliance. Three further patients were unable to complete the trial as per protocol because of lack of compliance after application of DPL. This left a total of 125 patients for final analysis. All treatment groups were well matched for age, gender, and weight (table 2).

Table 2 Ages, weights, and sex differences for the four study groups

\begin{tabular}{|c|c|c|c|c|c|c|c|c|}
\hline & \multicolumn{2}{|l|}{ A } & \multicolumn{2}{|l|}{ B } & \multicolumn{2}{|l|}{ C } & \multicolumn{2}{|l|}{ D } \\
\hline & DPL & Sham & DPL & Sham & DPL & Sham & DPL & Sham \\
\hline \multicolumn{9}{|l|}{ Back of hand } \\
\hline $\begin{array}{l}\text { Total (n) } \\
\text { (M/F) }\end{array}$ & $\begin{array}{l}10 \\
(4 / 6)\end{array}$ & $\begin{array}{l}5 \\
(2 / 3)\end{array}$ & $\begin{array}{l}13 \\
(8 / 5)\end{array}$ & $\begin{array}{l}5 \\
(4 / 1)\end{array}$ & $\begin{array}{l}10 \\
(5 / 5)\end{array}$ & $\begin{array}{l}5 \\
(2 / 3)\end{array}$ & $\begin{array}{l}10 \\
(2 / 8)\end{array}$ & $\begin{array}{l}5 \\
(3 / 2)\end{array}$ \\
\hline Age $(y)$, mean $(S D)$ & $9.3(1.6)$ & $7.4(0.5)$ & $8.9(2.2)$ & $7.2(2.2)$ & $7.8(2.1)$ & $8.0(2.5)$ & $8.0(2.3)$ & $6.0(0.7)$ \\
\hline Weight $(\mathrm{kg})$, mean (SD) & $23.7(4.7)$ & $23.7(4.7)$ & $31.2(10.3)$ & $23.7(4.3)$ & $30.2(9.9)$ & $30.5(10.7)$ & $30.0(9.1)$ & $26.4(5.6)$ \\
\hline \multicolumn{9}{|l|}{ Antecubital fossa } \\
\hline $\begin{array}{l}\text { Total }(n) \\
(\mathrm{M} / \mathrm{F})\end{array}$ & $\begin{array}{l}11 \\
(5 / 6)\end{array}$ & $\begin{array}{l}5 \\
(4 / 1)\end{array}$ & $\begin{array}{l}10 \\
(8 / 2)\end{array}$ & $\begin{array}{l}6 \\
(4 / 2)\end{array}$ & $\begin{array}{l}10 \\
(6 / 4)\end{array}$ & $\begin{array}{l}5 \\
(3 / 2)\end{array}$ & $\begin{array}{l}10 \\
13 / 7\end{array}$ & $\begin{array}{l}5 \\
(4 / 1)\end{array}$ \\
\hline Age $(y)$, mean (SD) & $8.8(2.0)$ & $9.4(2.9)$ & $7.6(1.7)$ & $7.8(2.1)$ & $8.4(2.5)$ & $8.6(1.5)$ & $8.0(2.2)$ & $7.8(1.3)$ \\
\hline Weight $(\mathrm{kg})$, mean (SD) & $33(9.8)$ & $36.3(19.4)$ & $26.9(5.4)$ & $30.8(15.7)$ & $27.4(8.4)$ & $28.9(4.9)$ & $26.4(5.7)$ & $27.3(2.3)$ \\
\hline
\end{tabular}


Table 3 Intensity of pain as measured by the Faces pain scale after cannulation at the antecubital fossa for DPL versus placebo

\begin{tabular}{llllll}
\hline & \multicolumn{2}{l}{ Faces score, median (range) } & & \multicolumn{2}{l}{ Differences between treatments } \\
\cline { 2 - 3 } Study group & Lignocaine & Sham & & Median (95\% CI) & Significance \\
\hline A & $2(0-4)$ & $1(0-6)$ & & $0(-2$ to 2$)$ & 0.77 \\
B & $0.5(0-3)$ & $1.5(1-6)$ & & $-1(-4$ to 0$)$ & 0.048 \\
C & $1(0-3)$ & $2(1-4)$ & & $-1(-2$ to 0$)$ & 0.12 \\
$D$ & $0(0-6)$ & $3(1-6)$ & & $-2.5(-5$ to 0$)$ & 0.019 \\
\hline
\end{tabular}

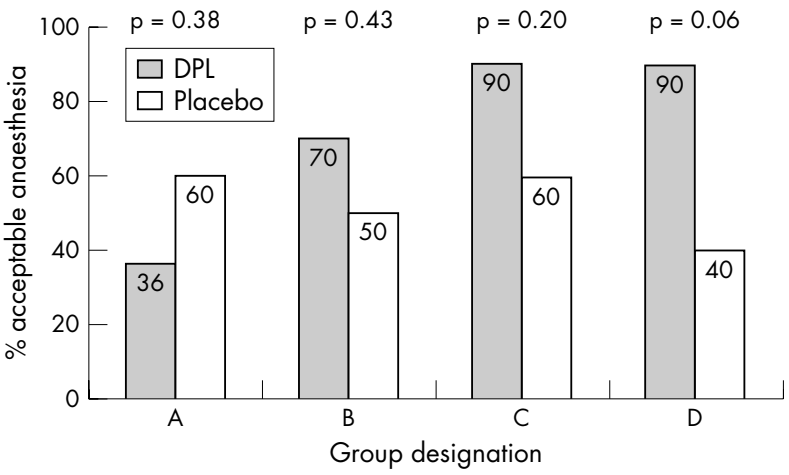

Figure 2 Percentage of patients with acceptable anaesthesia at the antecubital fossa, 3 minutes after application of DPL or placebo using self reported measures of pain from the Oucher pain scale.

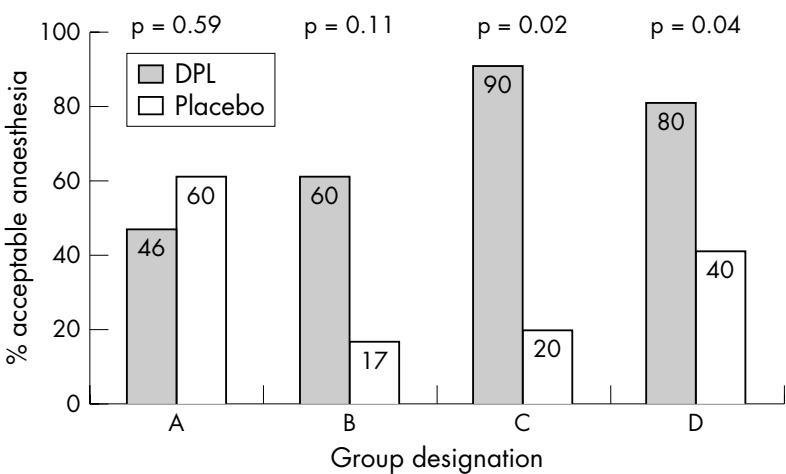

Figure 3 Percentage of patients pain free on cannulation at the antecubital fossa 3 minutes after application of DPL or placebo using the nurse's blinded pain assessment.

At the ACF site, there was a trend towards a higher percentage of acceptable pain scores after intravenous cannulation in the active compared to the sham treatments at higher activation pressures and lignocaine particle sizes at the ACF (fig 2), but this just failed to reach statistical significance $(\mathrm{p}=0.06$ with 40 bar activation pressure and 38-53 $\mu \mathrm{m}$ lignocaine particles). However, a comparison of pain intensity between the active and sham groups at the ACF using the Faces pain scale reached a significance level of 0.048 in group $\mathrm{B}$ and 0.019 in group D (table 3). Assessment of pain on cannulation by the blinded study nurse again showed significantly higher pain free cannulation $(\mathrm{p}<0.05)$ with DPL at higher pressures compared with the sham treatments (fig 3 ).

At the $\mathrm{BH}$, there was a trend towards DPL to be better than sham when administered to the $\mathrm{BH}$, but these differences were not statistically significant.

The device was well tolerated when deployed on the skin. Of the 128 children (both active and sham) who were treated with the device $102(80 \%)$ reported no pain; 21 of 85 patients in the active group (25\%) and five of 43 in the sham group
( $12 \%)$ reported mild or moderate pain. There was no trend towards increased pain on activation in patients who were in the groups receiving higher pressures or particle sizes. One patient receiving high pressure/low particle size lignocaine in the ACF was judged to have a Draize score of 3. All other patients had a Draize score of 2 or less. One patient reported itching of the hand after receiving lignocaine.

\section{DISCUSSION}

High velocity particles are capable of penetrating the epidermis and can deliver drugs systemically. ${ }^{10}$ We have adapted this technique with a prototype device (DPL) to determine if lignocaine particles can be embedded in the skin to produce rapid topical analgesia in children. The results show that at the ACF it is possible to reduce or eliminate the pain of cannulation better than a sham device and that the higher pressure of 40 bar is more effective than 30 bar. It is unclear from this study whether particle size affects the onset and quality of anaesthesia.

One of the confounding issues in this study was the low incidence and intensity of pain recorded by the children undergoing venous cannulation, and by the observing study nurse. In the sham group, over $50 \%$ of patients reported minimal or no pain (Oucher scores of 0,1 , or 2 ) on venous cannulation at either the ACF ( 14 of 21 ) or BH ( 10 of 21 ). This made it difficult to resolve differences between treatment and study groups and had not been expected. However, all cannulations were carried out by experienced paediatric anaesthetists and this group of patients did not have previous experience of hospital visits or blood taking procedures, which could explain the low pain scores in this group of patients. Generally, the self reporting pain scores agreed well with the nurse observer. However, on several occasions children who showed significant facial expressions of pain failed to score pain on their self reported assessment, and this may have influenced the results. While the primary outcome measure in this study was chosen to be self reporting measures of pain, a recent study in a similar group of patients, concluded that observed facial expression is a more reliable measure of pain on venepuncture than self reporting techniques. ${ }^{11}$ This suggests that when assessing pain in this age group, while both assessments are valuable, more emphasis should be put on pain assessment from the blinded observer than from the child.

The current prototype device failed to show benefit at the $\mathrm{BH}$ from either patient self evaluation or from the nurse observation. The most likely explanation is that the $\mathrm{BH}$ site has reduced sensitivity to noxious stimuli, while the increased thickness of the skin over the back of the hand would make penetration by lignocaine more difficult. Future devices will need to evaluate increased pressure or changes in particle size to improve lignocaine penetration. However, it is already clear that as pressure and particle size increases, the skin damage in terms of petechiae and surface bleeding also increases. In this study, only one patient had a Draize score of 3, but there was a trend towards skin trauma with higher pressures and larger particle sizes. 
One of the major fears of the child entering hospital is painful needle insertion. ${ }^{12}$ EMLA cream and Ametop cannot always be used effectively, either because they take too long to produce anaesthesia or because of failure to insert the cannula at the chosen site. The DPL prototype is effective at the ACF but further development is needed. To be widely accepted it will need to be effective at other cannulation sites without significant skin trauma. It could then become a valuable alternative to the current anaesthetic creams. The rapid onset of skin anaesthesia with DPL would allow an appropriate skin site to be selected and cannulated without delay when topical creams are ineffective, to achieve painless intravenous cannulation.

\section{Authors' affiliations}

A R Wolf, P A Stoddart, P J Murphy, Bristol Royal Hospital for Sick Children, St Michael's Hill, Bristol, UK

M Sasada, Bristol Dental Anaesthetic Clinic, Clifton, Bristol, UK

\section{REFERENCES}

1 Arendt-Neilson L, Bjerring P. Laser-induced pain for evaluation of local analgesia: a comparison of topical application (EMLA) and local anaesthetic injection (lignocaine). Anesth Analg 1988:67:115-23.

2 Lawson RA, Smart NG, Gudgeon AC, et al. Evaluation of amethocaine gel preparation for percutaneous analgesia before venous cannulation in children. Br J Anaesth 1995;75:282-5.
3 Romsing J, Henneberg SW, Walther-Larsen S, et al. Tetracaine gel vs EMLA cream for percutaneous anaesthesia in children. BrJ Anaesth 1999;82:637-8.

4 Koh JL, Fanurik D, Stoner PD, et al. Efficacy of parental application of eutectic mixture of local anaesthetics for intravenous insertion. Pediatrics 1999; 103:e79.

5 Sarphie D, Greenford J, Ashcroft JH, et al. Transdermal powdered delivery (TPD): in vivo determination of particle penetration depth and therapeutic efficacy. Proceedings of fourth percutaneous conference, $\mathrm{Vol}$ 4B. Cardiff: STS Publishing, 1996.

6 Beyer J, Aradine C. Content and validity of an instrument to measure young children's perception of the intensity of their pain. J Pediatr Nurs 1986; 1:386-95.

7 Bieri D, Reeve RA, Champion GD, et al. The faces pain scale for self assessment of the severity of pain experienced by children: development initial validation, and preliminary investigation for ratio scale properties. Pain 1990:41:139-50.

8 Draize JH. Dermal and eye toxicity tests. In: Principles and procedures for evaluating the toxicity of household substances. Washington, DC National Academy of Sciences, 1997:31-2.

9 SAS Institute Inc. SAS/STAT user's guide, version 6, Volume 2, 4th edn. Cary, NC: SAS Institute Inc., 1989.

10 Hickey PLC. Powder injection-a novel mode of drug delivery opening the way for new modes of therapy. Drug Delivery Systems and Sciences $2001 ; 1: 25-9$.

11 Goodenough TB, Perrott DA, Champion GD, et al. Painful pricks and prickle pains: is there a relation between children's ratings of venepuncture pain and parenteral assessments of usual reactions to other pains? Clin J Pain 2000;16:135-43.

12 Polkki T, Pietila AM, Rissanen L. Pain in children: qualitative research of Finish school aged children's experiences of pain in hospital. Int J Nurs Pract 1999;5:21-8

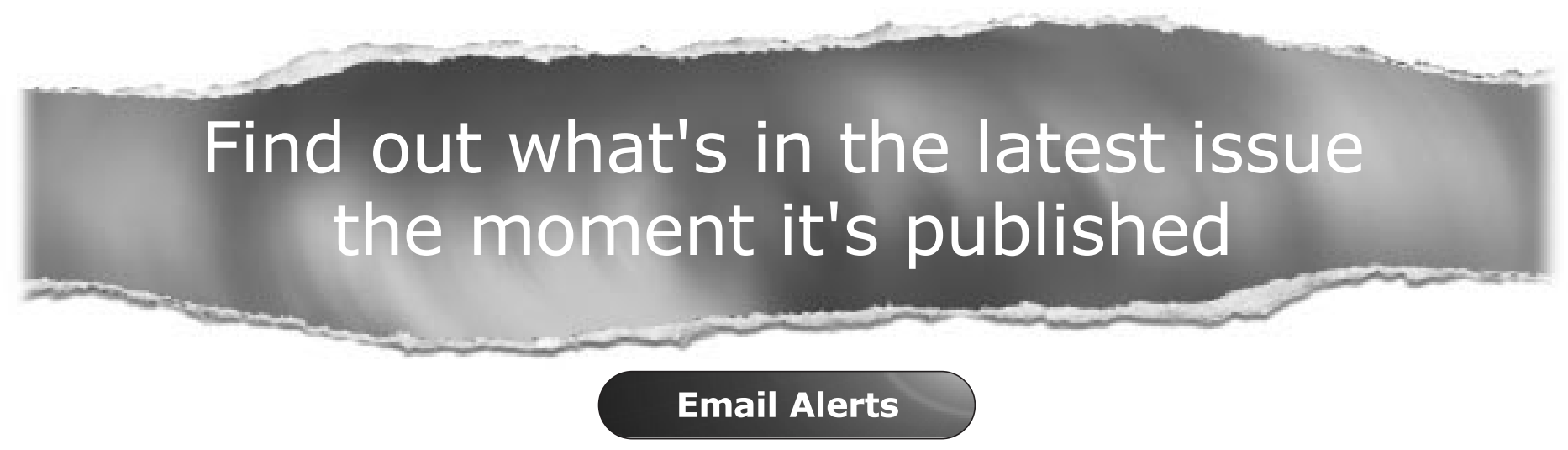

Sign up to receive the table of contents by email every month. You can select from three alerts: Table of Contents (full), TOC Awareness (notice only); Archives of Disease in Childhood related announcements.

\section{www.archdischild.com}

